\title{
Performance Analysis of ANN Training Algorithms to Detect the Magnetization Level in the Magnetic Core of a Welding Transformer
}

\author{
Rama Subbanna, S. ${ }^{1}$, Dr. Suryakalavarthi, M. ${ }^{2}$ \\ ${ }^{I}$ Department of Electrical and Electronics Engineering, St. Martins Engineering College, Hyderabad, India, \\ ${ }^{2}$ Department of Electrical and Electronics Engineering, Jawaharlal Nehru Technological University, \\ Hyderabad, India,
}

\begin{abstract}
The aim of this study is to perform an analysis of the different ANN methods employed to detect the saturation level in the magnetic core of a welding transformer. The magnetization level detector is a substantial component of a middle-frequency direct current (MFDC) resistance spot welding system (RSWS). The basic circuit of a resistance spot welding system consists of an input rectifier, an inverter, a welding transformer, and a full-wave rectifier which is mounted on the output of the welding transformer. The presence of unbalanced resistances of the transformer secondary windings and the difference in characteristics of output rectifier diodes can cause the transformers magnetic core to become saturated. This produces spikes in the primary current and finally leads to the over-current protection switch-off of the entire system. To prevent the occurrence of such a phenomena, the welding system control must detect and maintain the magnetic core saturation within certain limits. Previously, an Artificial Neural Network based detector was proposed to detect the saturation level. In this paper, we will take an in depth look at the different ANN methods that can be employed, analyze them and then decide which is the best method based on factors such as computational time, algorithm complexity, root mean square error and the gradient obtained. Three algorithms in total are evaluated including Resilient-Back propagation, Levenberg-Marquardt, Gradient-Descent. Based on the results obtained, the LevenbergMarquardt and the Resilient Back propagation were found to be the best algorithms but the Resilient Back propagation is preferred due to reasons such as computational time and algorithm complexity. In terms of RMSE both gave almost the same values. It is also shown that the Gradient Descent algorithm cannot be employed for this purpose.
\end{abstract}

Keywords: Transformers, hysteresis, welding, neural network applications, controllers, gradient descent algorithm, levenberg-marquardt algorithm, resilient back propagation algorithm, training methods.

\section{Introduction}

The aim of this work is to develop and evaluate the best possible ANN training method to detect the magnetization level in the magnetic core of a welding transformer. This is done by considering factors such as computational time, algorithm complexity, mean square error and the gradients of the ANNs. The magnetization level detector is a substantial component of a middle-frequency direct current (MFDC) resistance spot welding system (RSWS), where the welding current and the flux density in the welding transformer's magnetic core are controlled by two hysteresis controllers [1].

The resistance spot welding systems described in different realizations [2]-[5], are widely used in the automotive industry. Although the alternating or direct currents (dc) can be used for welding, this work focuses on the RSWS (Fig. 2) with dc welding current. The resistances of the two secondary windings R2, R3 and characteristics of the rectifier diodes, connected to these windings, can slightly differ. References [6]-[9] show that combination of these small differences can result in increased dc component in welding transformer's magnetic core flux density. It causes increasing magnetic core saturation with high impact on the transformer's primary current i1, where current spikes eventually appear, leading to the over-current protection switch-off of the entire system. However, the problematic current spikes can be prevented either passively [6] or actively [7][9].

When the current spikes are prevented actively, closed-loop control of the welding current and magnetic core flux density is required. Thus, the welding current and the magnetic core flux density must be measured. While the welding current is normally measured by the Rogowski coil [10], the magnetic core flux density can be measured by the Hall sensor or by a probe coil wound around the magnetic core. In the latter, the flux density value is obtained by analogue integration of the voltage induce in the probe coil [7]. Integration of the induced voltage can be unreliable due to the unknown integration constant in the form of remnant flux and drift in analogue electronic components. The drift can be kept under control by the use of closed-loop compensated analogue integrator [9]. 
An advanced, two hysteresis controllers based control of the RSWS, where current spikes are prevented actively by the closed-loop control of the welding current and flux density in the welding transformer's magnetic core, is presented in [9]. This solution requires measuring of the welding current, while instead of measured flux density only information about magnetization level in the magnetic core is required.

Some methods tested on welding transformer's magnetic core, that can be applied for magnetization level detection are presented in [7], [8]. All these methods require Hall sensor or probe coils which make them less interesting for applications in industrial RSWS, due to the relatively high sensitivity on vibrations, mechanical stresses and high temperatures. In order to overcome these problems, an ANN based magnetic core magnetization level detector was presented previously in [11]. Its only (single) input is the measured transformer's primary current. The ANN based magnetic core magnetization level detector is trained to recognize the waveform of the current spikes, which appear in the primary current when the magnetic core is approaching the saturated region. Upon detection of a spike, the ANN target signal makes it possible for the transformer supply voltage to change direction which also changes the magnetic flux density accordingly. This way, the system is controlled using the ANN detector and over-current protection switch-off is prevented.

In this paper, we will take an in-depth look at the different ANN methods employed and perform a comparative study of each and finally decide the best method based on the various factors and parameters involved.

\section{Artificial Neural Network}

[12] and [13] described the basic theories of ANN modelling as the following paragraphs. An ANN is a biologically inspired computational model formed from several of single units, artificial neurons, connected with coefficients (weights) which constitute the neural structure. They are also known as processing elements (PE) as they process information. Each PE has weighted inputs, transfer function and one output. PE is essentially an equation which balances inputs and outputs. There are many types of neural networks designed by now and new ones are invented every week but all can be described by the transfer functions of their neurons, by the training or learning algorithm (rule), and by the connection formula.

A single-layer neuron is not able to learn and generalize the complex problems. The multilayer perceptron (MLP) overcomes the limitation of the single-layer perceptron by the addition of one or more hidden layer(s) (Fig. 1). The MLP has been proven to be a universal approximator [14]. In Fig. 1, a feedforward multilayer perceptron network was presented. The arriving signals, called inputs, multiplied by the connection weights (adjusted) are first summed (combined) and then passed through a transfer function to produce the output for that neuron. The activation (transfer) function acts on the weighted sum of the neuron's inputs and the most commonly used transfer function is the sigmoid (logistic) function.

The way that the neurons are connected to each other has a significant impact on the operation of the ANN (connection formula). There are two main connection formulas (types): feedback (recurrent) and feed forward connection. Feedback is one type of connection where the output of one layer routes back to the input of a previous layer, or to same layer. Feed forward does not have a connection back from the output to the input neurons.

There are many different learning rules (algorithms) but the most often used is the Delta-rule or back propagation (BP) rule. A neural network is trained to map a set of input data by iterative adjustment of the weights. Information from inputs is fed forward through the network to optimize the weights between neurons.

Optimization of the weights is made by backward propagation of the error during training or learning phase. The ANN reads the input and output values in the training data set and changes the value of the weighted links to reduce the difference between the predicted and target (observed) values. The error in prediction is minimized across many training cycles (iteration or epoch) until network reaches specified level of accuracy. A complete round of forward-backward passes and weight adjustments using all input-output pairs in the data set is called an epoch or iteration. If a network is left to train for too long, however, it will be over-trained and will lose the ability to generalize.

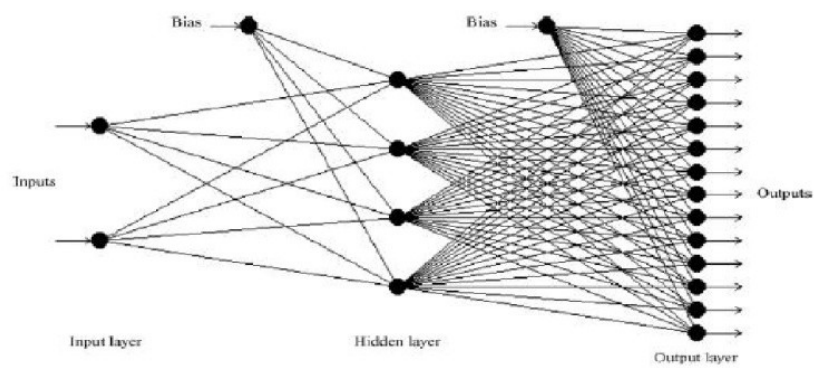

Fig. 1: Schematic representation of a multilayer perceptron feed forward network consisting of input, hidden and 
output layers.

The number of PEs per layer, as well as the number of layers, greatly influences the prediction abilities of the MLP. Too few of them hinder the learning process, and too many of them can depress the generalizing abilities of the MLP through over-fitting or memorization of the training data set.

In this study, we have focused on the learning situation known as supervised learning, in which a set of input/output data patterns is available. Thus, the ANN has to be trained to produce the desired output according to the examples.

In order to perform a supervised learning we need a way of evaluating the ANN output error between the actual and the expected output. A popular measure is the mean squared error (MSE) or root mean squared error (RMSE):

$$
\begin{aligned}
& \operatorname{MSE}=\underline{\Sigma(y i}-\text { oi })^{2} \\
& \mathrm{n} \\
& \mathrm{RMSE}=(\mathrm{MSE})^{1 / 2}
\end{aligned}
$$

Where $y_{\mathrm{i}}$ is the predicted value, $o_{\mathrm{i}}$ the observed value, and $\mathrm{n}$ is the number of data set.

As previously mentioned, there are numerous algorithms, which can be used for training ANNs. The following algorithms were used in this study to train ANNs:

\section{(1) Resilient Back propagation:}

Multilayer networks typically use sigmoid transfer functions in the hidden layers. These functions are often called "squashing" functions, because they compress an infinite input range into a finite output range. Sigmoid functions are characterized by the fact that their slopes must approach zero as the input gets large. This causes a problem when you use steepest descent to train a multilayer network with sigmoid functions, because the gradient can have a very small magnitude and, therefore, cause small changes in the weights and biases, even though the weights and biases are far from their optimal values.

The purpose of the resilient back propagation (Rprop) training algorithm is to eliminate these harmful effects of the magnitudes of the partial derivatives. Only the sign of the derivative can determine the direction of the weight update; the magnitude of the derivative has no effect on the weight update. The size of the weight change is determined by a separate update value. The update value for each weight and bias is increased by a factor delt_inc whenever the derivative of the performance function with respect to that weight has the same sign for two successive iterations. The update value is decreased by a factor delt_dec whenever the derivative with respect to that weight changes sign from the previous iteration. If the derivative is zero, the update value remains the same. Whenever the weights are oscillating, the weight change is reduced. If the weight continues to change in the same direction for several iterations, the magnitude of the weight change increases. A complete description of the Rprop algorithm is given in [15].

\section{(2) Gradient Descent:}

This algorithm is one of the most popular training algorithms in the domain of neural networks. It works by measuring the output error, calculating the gradient of this error, and adjusting the ANN weights (and biases) in the descending gradient direction. Hence, this method is a gradient descent local search procedure [16]. This algorithm includes different versions such as: (2a) standard or incremental back propagation (IBP): the network weights are updated after presenting each pattern from the learning data set, rather than once per iteration [17]; (2b) batch back propagation (BBP): the network weights update takes place once per iteration, while all learning data pattern are processed through the network [18]; (2c) quick propagation (QP): QP is a heuristic modification of the back propagation algorithm. It is proved much faster than IBP for many problems. $\mathrm{QP}$ is also defined as: mixed learning heuristics without momentum, learning rate optimized during training [19].

\section{(3) Levenberg-Marquardt:}

The Levenberg-Marquardt algorithm was designed to approach second-order training speed without having to compute the Hessian matrix [20]. When the performance function has the form of a sum of squares (as is typical in training feed forward networks), then the Hessian matrix can be approximated as

$\mathrm{H}=\mathrm{J}^{\mathrm{T}} \mathbf{J}$

and the gradient can be computed as $\mathrm{g}=\mathrm{J}^{\mathrm{T}} \mathrm{e}$

where $J=$ Jacobian matrix, which contains first derivatives of the network errors with respect to the 
weights and biases, and $\boldsymbol{e}=$ vector of network errors. The Jacobian matrix can be computed through a standard back-propagation technique that is much less complex than computing the Hessian matrix.

$$
X_{k+1}=X_{k}-\left[J^{T} J+\mu I\right]^{-1} J^{T} e
$$

When the scalar $\mu$ is zero, this is just Newton's method, using the approximate Hessian matrix. When $\mu$ is large, this becomes gradient descent with a small step size. Newton's method is faster and more accurate near an error minimum, so the aim is to shift

To ward Newton's method as quickly as possible. Thus, $\mu$ is decreased after each successful step (reduction in performance function) and is increased only when a tentative step would increase the performance function. In this way, the performance function will always be reduced at each iteration of the algorithm. The application of Levenberg-Marquardt to neural network training is described elsewhere in [21].

\section{Dynamic Model Of The RSWS}

The RSWS, shown in Fig. 2, consists of an input rectifier, an H-bridge inverter, a single phase transformer and a full-wave output rectifier [9]. The three-phase alternating current (ac) voltages u1, u2, u3, supplied from the electric grid, are rectified in the input rectifier in order to produce the direct current (dc) bus voltage. This voltage is used in the H-bridge inverter, where different switching patterns and modulation techniques can be applied, to generate ac voltage $\mathrm{u}_{\mathrm{H}}$, required for supply of the welding transformer. The welding transformer has one primary and two secondary windings. They are marked with indices 1,2 and 3, respectively. The currents, the number of turns, the resistance and the leakage inductances of the primary and two secondary welding transformer's windings are denoted by $i_{1}, i_{2}, i_{3}, N_{1}, N_{2}, N_{3}, R_{1}, R_{2}, R_{3}$, and $L \sigma_{1}, L \sigma_{2}, L \sigma_{3}$.

The effects of the eddy current losses are accounted by the resistor $R_{\mathrm{Fe}}$. $\mathrm{R}_{1}$ and $\mathrm{L}_{1}$ are the resistance and inductance of the load. The output rectifier diodes $\mathrm{D}_{1}$ and $\mathrm{D}_{2}$ are connected to both transformers' secondary coils. They generate the de welding current $i_{1}$ which has a dc value a few times higher than the amplitudes of ac currents i2 and i3 that appear in the transformer's secondary coils without the rectifier diodes.

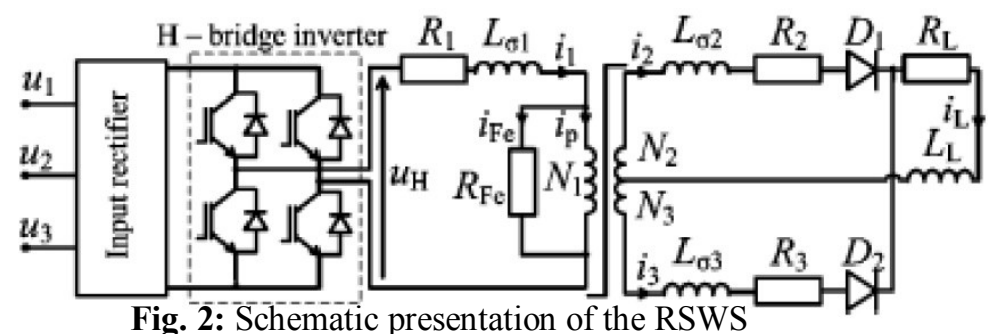

The supply voltage of the primary coil of the transformer could be generated in different ways. In the existent system, widely spread in the automotive industry, this voltage is generated by the H-bridge inverter applying pulse width modulation (PWM) at switching frequency of $f=1 \mathrm{kHz}$.

Within the welding system, the resistances of the secondary windings $R 2, R 3$ and the characteristics of the rectifier diodes could be slightly different. These differences can cause DC component in welding transformer's iron core flux density, which causes increasing iron core saturation with the essential impact on the transformer's primary current $i 1$, where current spikes appear, leading to the over-current protection switchoff of the entire system. Aforementioned phenomena could be confirmed by the appropriate dynamic model of the complete resistance spot welding system. In this work the model is built with the programme package Matlab/Simulink based on the schematic presentation shown in Fig. 2 and the following set of equations (6)(14). The results and the values obtained from this dynamic model are provided as inputs to each of the Artificial Neural Networks employed later.

$u_{\mathrm{H}}=R_{1} i_{1}+L \sigma_{1}\left(d i_{1} / d t\right)+N_{1}(d \varphi / d t)$

$0=R_{2} i_{2}+L \sigma_{2}\left(d i_{2} / d t\right)+N_{2}(d \varphi / d t)+d i p_{1}+R_{\mathrm{L}} i_{\mathrm{L}}+L_{\mathrm{L}}\left(\mathrm{d}\left(i_{2}+i_{3}\right) / d t\right)$

$0=R_{3} i_{3}+L \sigma_{3}\left(d i_{3} / d t\right)-N_{3}(d \varphi / d t)+d i p_{2}+R_{L} i_{\mathrm{L}}+L_{\mathrm{L}}\left(d\left(i_{2}+i_{3}\right) / d t\right) \ldots$

$N_{l} i_{\mathrm{p}}+N_{2} i_{2}-N_{3} i_{3}=H(B) l_{\mathrm{ic}}+2 \delta B / \mu_{0}$

$i_{\mathrm{L}}=i_{2}+i_{3}$

$i_{1}=i_{\mathrm{Fe}}+i_{\mathrm{P}}$

$i_{\mathrm{Fe}}=N_{1}(d \varphi / d t) / R_{\mathrm{F}}$

$\varphi=B A_{\mathrm{F}}$

$\theta=N_{1} i_{1}+N_{2} i_{2}-N_{3} i_{3}$

In set of equations (6) - (14) $\varphi$ stands for magnetic flux, dip 1 and dip 2 are nonlinear characteristics of 
the output rectifier diodes $D 1$ and $D 2, H(B)$ is the magnetizing curve of the iron core material, $\delta$ is the air gap, $B$ is the iron core flux density, $\mu_{0}$ is the permeability of the vacuum, lic is the average length of the magnetic flux line in the iron core, $A_{\mathrm{Fe}}$ is the cross-section of the transformer's iron core and $\theta$ is the magneto-motive force. Parameters that appear in (6) - (13) are shown in Table 1 and in Table 2. These are generally the values employed. The Dynamic Model employed in this work also utilizes the same values presented below:

Table 1: Parameters of RSWS Dynamic Model

\begin{tabular}{|c|c|c|}
\hline Parameter & Value & Unit \\
\hline $\mathrm{AFe}$ & 0.001385 & $m^{2}$ \\
\hline$\delta$ & 10 & $\mu \mathrm{m}$ \\
\hline lic & 0.313 & $\mathrm{~m}$ \\
\hline $\mathrm{f}$ & 1000 & $\mathrm{~Hz}$ \\
\hline R1 & 0.01357 & $\Omega$ \\
\hline R2 & 20 & $\mu \Omega$ \\
\hline R3 & 20 & $\mu \Omega$ \\
\hline RL & 100 & $\mu \Omega$ \\
\hline $\mathrm{L} \sigma 1$ & 0.035 & $\mathrm{mH}$ \\
\hline $\mathrm{L} \sigma 2$ & 1 & $\mathrm{nH}$ \\
\hline Lo3 & 1 & $\mathrm{hH}$ \\
\hline LL & 1 & $\mu \mathrm{H}$ \\
\hline N1 & 55 & \\
\hline N2 & 1 & \\
\hline N3 & 1 & 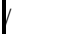 \\
\hline
\end{tabular}

Table 2: Nonlinear characteristics of the output rectifier diodes $D 1$ - (dip1) and D2 - (dip2)

\begin{tabular}{|l|l|l|l|}
\hline & dip1-i(A) & dip2-i(A) & dip2-u(V) \\
\hline 0 & 0 & 0 & 0 \\
\hline 0.003 & 0.6 & 0.011 & 0.6 \\
\hline 0.014 & 0.65 & 0.053 & 0.65 \\
\hline 0.059 & 0.7 & 0.25 & 0.7 \\
\hline 0.247 & 0.75 & 1.17 & 0.75 \\
\hline 1.05 & 0.8 & 5.52 & 0.8 \\
\hline 4.43 & 0.85 & 25.9 & 0.85 \\
\hline 18.75 & 0.9 & 121.5 & 0.9 \\
\hline 79.3 & 0.95 & 570 & 0.95 \\
\hline 335 & 1 & 2675 & 1 \\
\hline 1418 & 1.05 & 12555 & 1.05 \\
\hline 6000 & 1.1 & 58912 & \\
\hline
\end{tabular}




\begin{tabular}{|l|l|l|l|} 
& & & \\
\hline 25378 & 1.15 & 400416 & 1.15 \\
\hline 107334 & 1.2 & 1297043 & 1.2 \\
\hline
\end{tabular}

The results of simulations, obtained by the dynamic model of the RSWS, show that small difference in resistances R2, R3 and in characteristics of the rectifier diodes D1 and D2 can cause unbalanced time behaviour of the magnetic core flux density B and the current spikes in the primary current i1, shown in Fig.3. The a) graph in Fig. 3 shows the variation of flux with time. It can be seen that after a certain point, the flux begins to rise and goes on to reach the saturation levels. The b), c) and d) graphs in Fig. 3 show the variation of primary current in different time scales. As the core begins to saturate and the flux increases beyond the limits, the current spikes start to appear in the welding transformers primary current. From Fig. 3(c), it can be clearly seen that the spikes begin to appear at around $0.075 \mathrm{~s}$. These spikes start to gradually increase with time and finally become sufficient to cause over-current protection switch-off of the entire welding system. In order to prevent this, we must first detect these spikes using an appropriate method and then suppress the detected spikes.

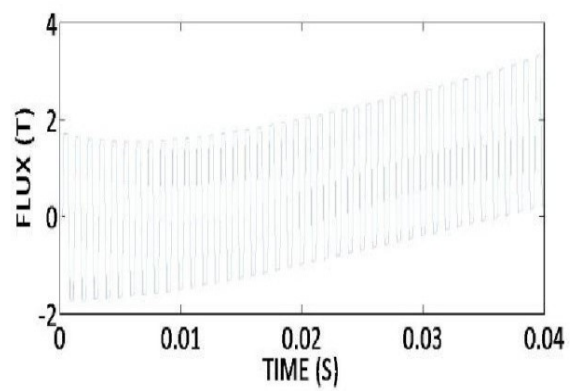

3(a): Time behaviour of Flux B

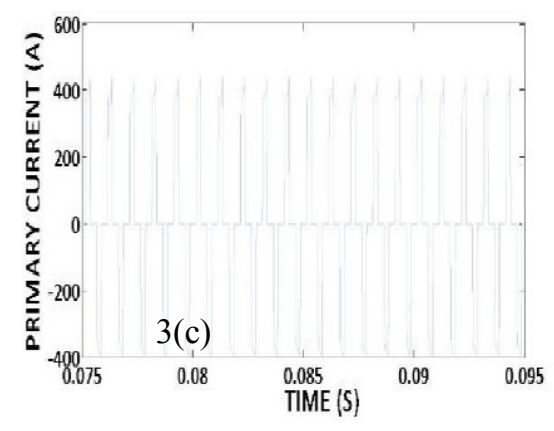

Fig. 3( b),3(c),
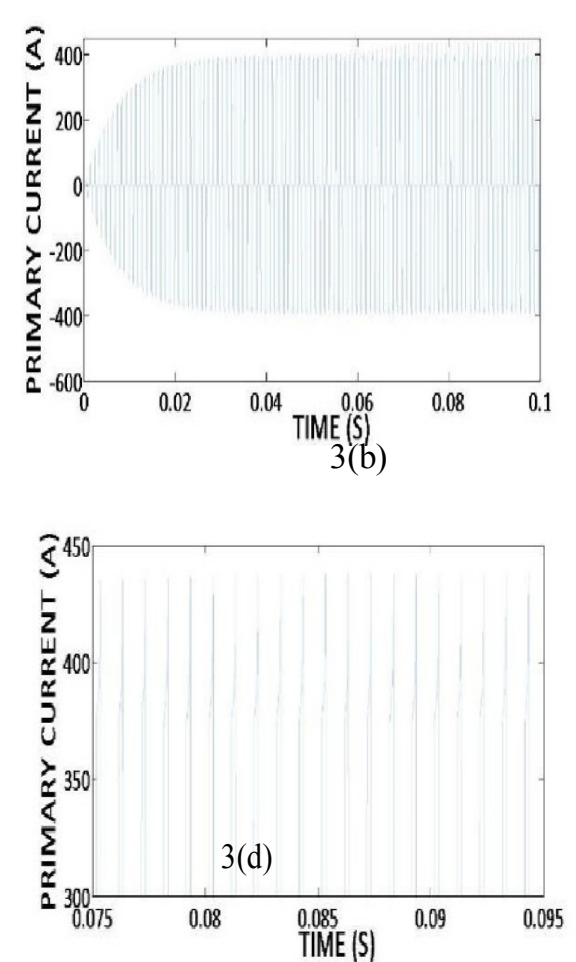

3(d): Time behaviour of primary current $i_{1}$

\section{Results and Discussion}

In each of the mentioned ANN training algorithms, the only input to the ANN is the primary current of the welding system obtained from the above established dynamic model. Based on whether there is a spike occurring or not, the output of the ANN is obtained. Whenever, a spike is detected, the output of the ANN is set to 1. Then, a Hysteresis Controller which operates in conjunction with the ANN based detector performs a closed-loop control of the transformers' primary voltage. With this, the flux and hence the primary current are reduced and brought back within their operating limits. Whenever a spike is detected, the transformers' primary voltage reverses its polarities and hence the flux changes its direction towards the other peak. The simulations for each of the training algorithms of the ANN were performed by considering various architectures. Also, the total number of samples of input and output were around 4800. In this paper, we will present the results obtained for up to 1100 of these samples. From the simulations, the two best architectures for the ANNs were found be three layered networks with 30 neurons in the input, 7 neurons in the hidden, one neuron in the output layer and 50 neurons in the input, 8 neurons in the hidden and one neuron in the output layer respectively. The results of the learning signals of the ANNs for each of the specified algorithms and architecture are shown below. Alongside these, the expected output signal is also shown. 

welding Transformer

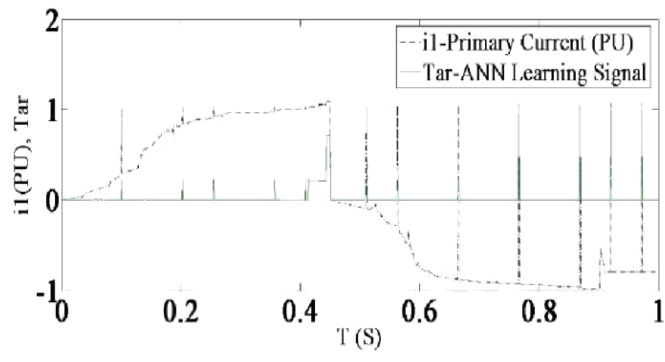

Fig. 4(a): Learning Signal of ANN with RBP Algorithm Algorithm with 30-7-1 architecture

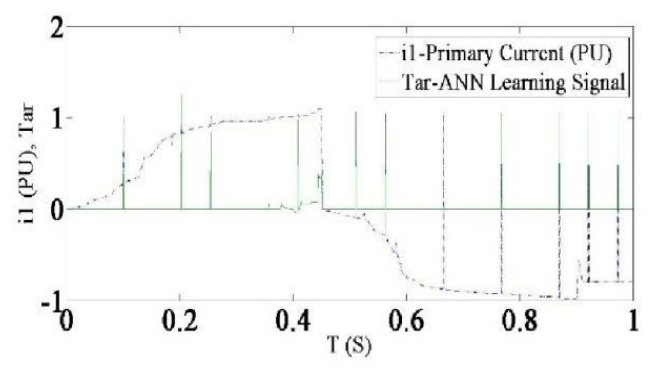

Fig. 4(c): Learning Signal of ANN with LM Algorithm Algorithm with 30-7-1 architecture

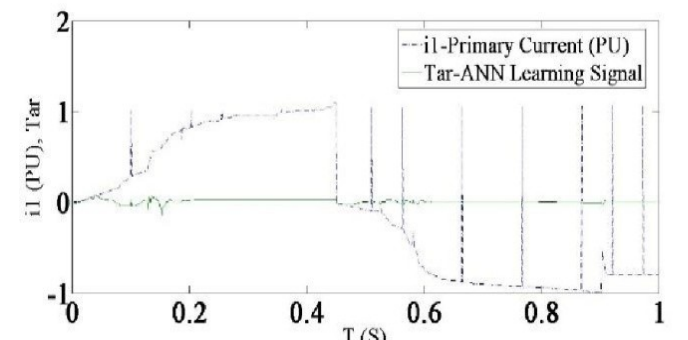

Fig. 4(e): Learning Signal of ÂNN with GD Algorithm Algorithm with 30-7-1 architecture

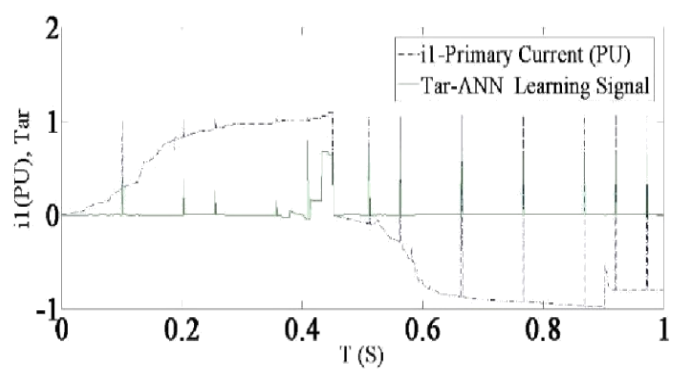

Fig. 4(b): Learning Signal of ANN with RBP with 50-8-1 architecture

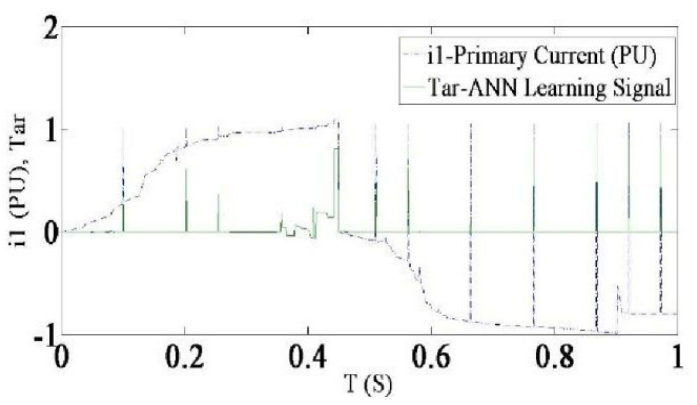

Fig. 4(d): Learning Signal of ANN with LM with 50-8-1 architecture

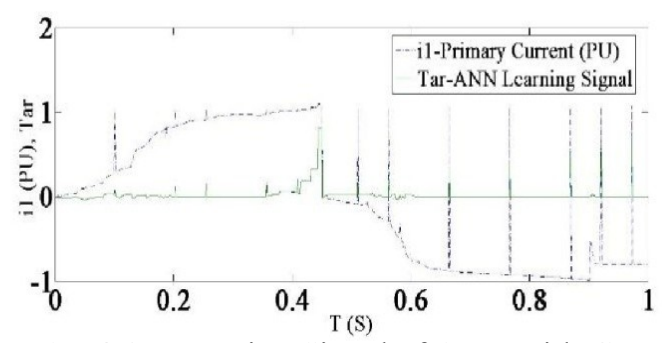

Fig. 4(f): Learning Signal of ANN with GD with 50-8-1 architecture

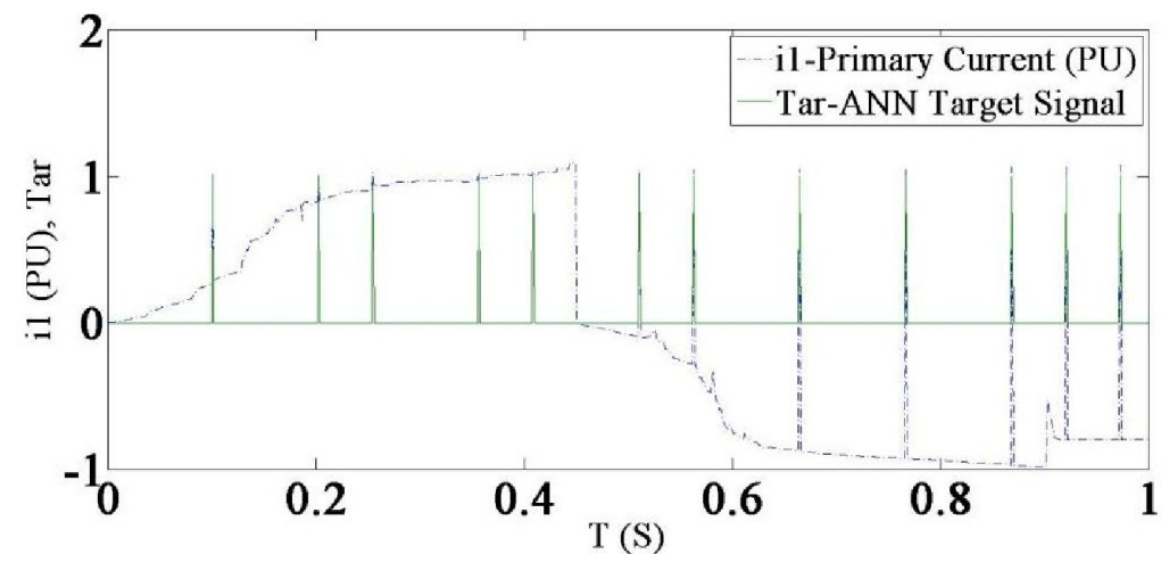

Fig. 4(g): ANN Expected Target Signal obtained after completion of all the training cycles for any of the above mentioned algorithms

From the above graphs it is clear that the ANN employed with the Levenberg-Marquardt algorithm produces the best results. While the results of Resilient Back propagation Algorithm are good enough and its 
results are relatively close to the Levenberg-Marquardt algorithm, the results of Gradient Descent algorithm are very poor and hence, it is clear that Gradient Descent cannot be employed for this purpose. The order of the best possible algorithms on the basis of the above learning signals is Levenberg-Marquardt $>$ Resilient

Backpropagation $>$ Gradient Descent. However, the Resilient Back propagation is comparatively easier to employ considering factors such as time taken for the training process and the relative complexities of each of the algorithms. The tables (1) and (2) below show the training times, gradients and the root mean squared errors for each of the algorithms for the architectures 30-7-1 and 50-8-1 respectively.

Table. 3: Table depicting a comparison of training times (s), RMSE values and gradients for the three algorithms for an ANN with the architecture 30-7-1

\begin{tabular}{|c|c|c|c|}
\hline $\begin{array}{c}\text { TRAINING } \\
\text { ALGORITHM }\end{array}$ & TRAINING TIME (sec) & RMSE & GRADIENT \\
\hline Resilient Backpropagation & 98 & 0.1018 & 0.34436 \\
\hline Levenberg-Marquardt & 503 & 0.0641 & 0.11137 \\
\hline Gradient Descent & 103 & 0.1890 & 0.0021649 \\
\hline
\end{tabular}

Table. 4: Table depicting a comparison of training times (s), RMSE values and gradients for the three algorithms for an ANN with the architecture 50-8-1

\begin{tabular}{|c|c|c|c|}
\hline TRAINING ALGORITHM & TRAINING TIME (sec) & RMSE & GRADIENT \\
\hline Resilient Backpropagation & 118 & 0.1176 & 0.0040654 \\
\hline Levenberg-Marquardt & 983 & 0.0934 & 0.0062879 \\
\hline Gradient Descent & 120 & 0.1118 & 0.0013543 \\
\hline
\end{tabular}

\section{Conclusion}

The aim of this work is to present the best possible ANN training algorithm and architecture employed to detect the magnetization level in the magnetic core of a welding transformer. The best possible method is determined by considering factors such as computation time (training time), algorithm complexity, RMSE values and the gradients obtained. Based on the extensive analysis performed and the results obtained, the following conclusions are made:

1) Levenberg-Marquardt algorithm gave the least RMSE values and the output signal obtained was closest to the target signal required.

2) The Gradient Descent algorithm gave the worst RMSE and the output signal obtained was far away from the required target signal.

3) The Resilient Back propagation algorithm gave RMSE values close to the Levenberg-Marquardt algorithm and the output signal obtained was pretty close to the required target signal.

4) The neural network architecture 30-7-1 was found to be the best possible architecture for the purpose.

Considering all the factors such as RMSE, computational time, algorithm complexity and the gradients obtained, it is concluded that the Resilient Back propagation algorithm is the most suitable method that can be employed to detect the magnetization level in the magnetic core of welding transformer.

\section{References}

[1] K. Deželak, J. Pihler, G. Štumberger, B. Klopčič and D. Dolinar, “Artificial Neural Network Applied for Detection of Magnetization Level in the Magnetic Core of a Welding Transformer,” IEEE Transactions on Magnetics, vol. 46, no. 2, pp. 634637, February 2010.

[2] W. Li, E. Feng, D. Cerjanec, and G. A. Grzazinski, "A comparison of AC and DC resistance welding of automotive steels," in Sheet Metal Welding Conf. XI, Sterling Heights, MI, May 2004.

[3] A.Canova, G. Gruosso, and B. Vusini, "Electromagnetic modelling of resistance spot welding system," in ISEF 2007, XIII Int. Symp. Electromagnetic Fields in Mechatronics, Czech Republic, 2007.

[4] B. M. Brown, "A comparison of AC and DC resistance welding of automotive steels," Welding J., vol. 66, no. 1, pp. 18-23, Jan. 1987.

[5] W. Li, D. Cerjanec, and G. A. Grzadzinski, "A comparative study of single AC and multiphase DC resistance spot welding," ASME Trans. J. Manuf. Sci. Eng., vol. 127, no. 3,pp. 583-889, 2005.

[6] B. Klopčič, G. Štumberger, and D. Dolinar, "Iron core saturation of a welding transformer in a medium frequency resistance spot welding system caused by the asymmetric output rectifier characteristics," in Conf. Rec. IAS 2007 Annu. Meeting, New Orleans, LA, Sep. 2007, pp. 2319-2326.

[7] K. Deželak, B. Klopčič, G. Štumberger, and D. Dolinar, "Detecting saturation level in the iron core of a welding transformer in a resistance spot-welding system,” J. Magn. Magn. Mater. vol. 320, no. 20, pp. 878-883, Oct. 2008.

[8] K. Deželak, G. Štumberger, B. Klopčič, D. Dolinar, and J. Pihler, "Iron core saturation detector supplemented by an artificial neural network," Prz. Elektrotech., vol. 84, no. 12, pp. 157-159, 2008.

[9] B. Klopčič, D. Dolinar, and G. Štumberger, "Advanced control of a resistance spot welding system," IEEE Trans. Power Electron., vol. 23, no. 1, pp. 144-152, Jan. 2008.

[10] D. J. Ramboz, "Machinable Rogowski coil, design, and calibration,” IEEE Trans. Instrum. Meas., vol. 45, no. 2, Apr. 1996. 
[11] Rama Subbanna,S., Kamalakar,K.S.K., Dr.Suryakalavathi,M., “Artificial Neural network for the detection of Magnetization level in the magnetic core of a welding Transformer",IJAER,Vol8, no.6, pp.56-60, 2013.

[12] Bourquin, J., Schmidli, H., Hoogevest, P.V., Leuenberger, H., 1997b. "Ba sic concepts of artificial neural networks (ANN) modeling in the application to pharmaceutical development." Pharm. Dev. Tech. 2, 95-109.

[13] Agatonovic-Kustrin, S., Beresford, R., 2000. "Basic concepts of artificial neural network (ANN) modelling and its application in pharmaceutical research.” J. Pharm. Biomed. Anal. 22, 717-727.

[14] Cybenko, G., 1989. “Approximation by superposition of a sigmoidal function.” Math. Control Signals Syst. 2, 303-314.

[15] Riedmiller $M$ \& Braun $H$, " A direct adaptive method for faster back propagation learning: The RPROP algorithm," Proc. IEEE Int Conf Neural Networks, 1993.

[16] Rumelhart, D., Hinton, G., Williams, R., 1986. "Learning representations by back propagation errors.” Nature 323, 533-536.

[17] Freeman, J.A., Skappura, D.M., 1991. "Neural Networks Algorithms, Applications and Programming Techniques." AddisonWesley, Houston, pp. 12-105.

[18] Hagan, M.T., Demuth, H.B., Beale, M.H., 1996. "Neural Network Design.” PWS, Boston.

[19] Fahlman, S.E., 1988. Faster-learning variations on back-propagation: an empirical study. In: Proceedings of the 1988 Connectionist Models Summer School.

[20] More, J.J, in Numerical Analysis, edited by Watson, G.A, Lecture Notes in Mathematics 630, (Springer Verlag, Germany) 1997, 105-116.

[21] Hagan, M.T., Menhaj, M.B., 1994. Training feed forward networks with the Marquardt algorithm. IEEE Trans. Neural Netw. 5, 989-993.

\section{Authors Bibliography}

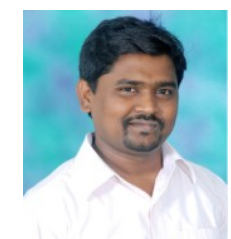

Rama Subbanna, S. graduated from Jawaharlal Nehru Technological University in the year 2003. M.Tech from Jawaharlal Nehru Technological University, Hyderabad, in the year 2006. Currently, He is pursuing Ph.D from Jawaharlal Nehru Technological University, Anantapur, India. Presently, he is working as Assistant Professor in St.Martin's Engineering College. His research includes Power Systems, Magnetic Materials, Controllers, ANN Techniques and Fuzzy Logic Methods.

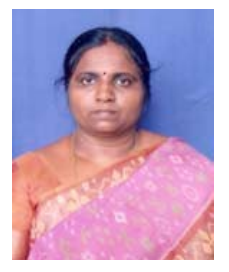

Dr.Suryakalavathi, M. graduated from S.V.University, Tirupati in the year 1988 . M.Tech from S.V.University, Tirupati in the year 1992. Ph.D from J.N.T.University, Hyderabad in the year 2006 and Post Doctoral from CMU, USA. She is presently professor of Electrical and Electronics Engineering department, J.N.T.U.College of Engineering, Hyderabad. She presented more than 50 research papers in various national and international conferences and Journals. Her research area includes High Voltage Engineering, Power Systems and Artificial Intelligence Methods. 